\section{Beads, bacteria and actin}

\section{Joseph W. Sanger and Jean M. Sanger}

ONCE again beads have been used to trick Mother Nature into revealing how actin moves things around on the cell surface $^{1,2}$. On page 515 of this issue ${ }^{2}$, Forscher and his collaborators illustrate how polycationic beads, which bind to the dorsal surface of nerve cell growth cones, can induce actin filament bundles, termed inductopodia, to form inside the cells. Not only can the extracellular beads induce intracellular actin filament assembly, but the beads associated with the filament bundles can then move along the cell surface. This striking phenomenon can be appreciated best by video-enhanced microscopy, as the authors showed at the meeting of the American Society for Cell Biology in December of last year.

Actually, two types of movement can be observed when beads are applied to the growth cone cell surface: a retrograde motility (away from the growth cone towards the nucleus), at the rate $1-6 \mu \mathrm{m} \mathrm{min} \operatorname{mat}^{-1}$, that is coupled to the submembranous, filamentous (F-) actin network ${ }^{1}$; and the newly identified motility coupled to the assembly of inductopodia (see figure) ${ }^{2}$. The trajectories of beads associated with elongating inductopodia can be straight or curved, and can be at any angle to retrograde flow.

The highest velocities occur when the beads move parallel to and in the same direction as retrograde flow. Subtraction of the retrograde F-actin flow rate from the total rate of bead motility shows that the bead-induced rate averages $10 \mu \mathrm{m}$ $\mathrm{min}^{-1}$ regardless of the angle of the path of movement. A requirement for continuing actin polymerization was shown by treating the beaded cells with cytochalasin, which disrupts F-actin. Within 15 seconds inductopodia elongation ceased, and both beads and inductopodia moved rearward in synchrony with the retracting cortical F-actin. As soon as cytochalasin was washed out, inductopodia growth and bead motility resumed. Coating the polycationic beads with a non-specific protein such as albumin also inhibited inductopodia formation but not the bead motility associated with retrograde flow.

The similarity of the bead-induced motility to that of some infectious bacteria such as Listeria ${ }^{3,4}$ and Shigella ${ }^{5,6}$ is as striking as the bead motility itself. These bacteria are engulfed by host cells but escape an unpleasant fate in the acidic environment of the phagolysosome by entering the infected cells' cytoplasm. In both systems, bead and bacterium, actin is recruited to polymerize into

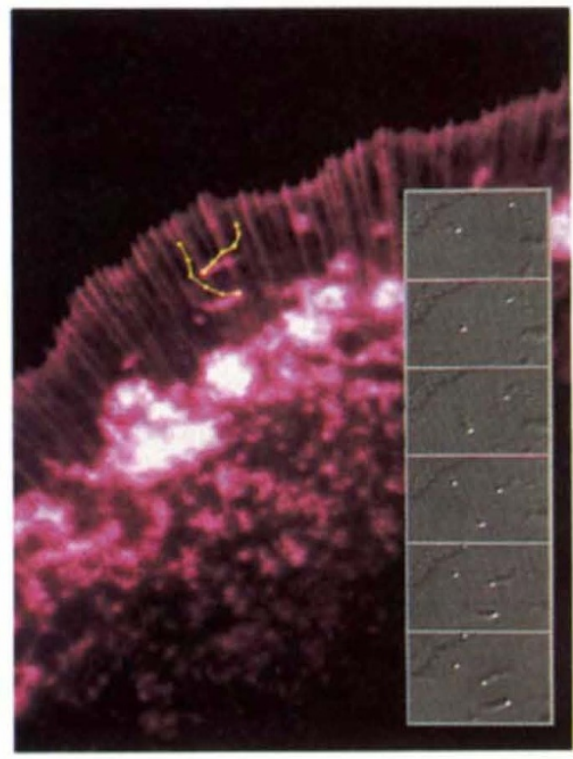

Polycationic beads in motion on the surface of a growth cone cell. The electronically enhanced video sequence (right, 10-second intervals) illustrates the movements of 240 $\mathrm{nm}$-diameter beads on the membrane surface. The two lower beads trigger submembranous actin filament assembly which is tightly coupled to lateral bead translocation. The background image shows the F-actin structure in this growth cone, demonstrating that the comet tail-like protrusions behind beads result from accumulation of F-actin. Corresponding bead positions over time are marked in yellow for the two lower beads in the video sequence. (IIlustration courtesy of P. Forscher.)

tail-like appendages ${ }^{6,7}$ and motility is inhibited reversibly by cytochalasin ${ }^{3}$. The trajectories and rates of motility are also remarkably similar ${ }^{3,4}$. The actin filaments in the bacterial tails are arranged with their fast-growing, or barbed ends, proximal to the bacteria ${ }^{8}$, and it is at this juncture where actin monomers insert as the tails elongate ${ }^{4}$. When a region of the tail is marked with fluorescent actin, the marked region remains stationary as the bacterium moves forward ${ }^{9}$. These data are consistent with a model in which the force of actin polymerization propels the bacteria forward ${ }^{3,4}$, although a role for myosin has not been ruled out entirely. The questions of both actin filament polarity and the actual site of actin polymerization are yet to be determined for inductopodia.

Whether or not actin-nucleating proteins are involved in either system is not known; such proteins promote the polymerization of actin monomers into filaments. But whereas the bacteria might synthesize them, clearly the beads do not. The binding of the beads to the cell membrane must initiate a transmembrane signal that enables nucleating proteins to localize at the binding site. Is it possible that, once they have entered the host cell, the bacteria in some way mimic the transmembrane complex and compete for the cells' actin-nucleating proteins?

The new paper by Forscher et al. ${ }^{2}$ may also point to a fresh way to look at another group of infectious bacteria that can histopathologically insult our intestines. When strains of enteropathogenic Escherichia coli attach to the intestine, the microvillous lining, so important in absorption, is lost in a process termed effacing ${ }^{10}$. It is not known how this process occurs. The bacteria normally do not enter the cell but are thought to remain firmly attached to the cell membrane. There is intriguing evidence that tufts of filaments project into the cytoplasm from the attachment sites ${ }^{10}$. Could these filaments be actin? And is it possible that these dysentery-causing bacteria move along the intestinal cell surfaces inducing the loss of the microvilli by appropriating their supply of actin? Cell biologists with intestinal fortitude might look into these questions.

Understanding how actin and other proteins are recruited to a particular region of the cell's plasma membrane remains a tough problem. This process is central to the formation of cleavage furrows, synaptosomes, myofibrils and stress fibres, and the bead experiments by Forscher et $a l^{2}$ suggest a way to analyse it - isolation of the beads with their inductopodia or Listeria with their actin tails may provide the means to isolate and identify the proteins involved. It is probably healthier to work with beads, but anyone who looks at Listeria travelling through infected cells will soon be seduced into considering the bacterial system.

Joseph W. Sanger and Jean M. Sanger of the Pennsylvania Muscle Institute are in the Department of Cell and Developmental Biology, University of Pennsylvania School of Medicine, Philadelphia, Pennsylvania 19194-6058, USA.

1. Forscher, P. \& Smith, S. J. J. Cell Biol. 107, 15051516 (1.988)

2. Forscher, P., Lin, C.H. \& Thompson, C. Nature 357 515-518 (1992).

3. Dabiri, G.A., Sanger, J.M., Portnoy, D.A. \& Southwick, F.S. Proc. natn. Acad. Sci. U.S.A. 87, 6068-6072 (1990).

4. Sanger, J.M., Sanger, J.W. \& Southwick, F.S. Infect Immun. (submitted).

5. Ogawa, H., Nakamura, A. \& Nakaya, R. Jap. J. med Sci. Biol. 21, 259-273 (1968).

6. Bernardini, M.L., Mounier, J., d'Hauteville, H., CoquisRodon, M. \& Sansonetti, P. J. Proc. natn. Acad. Sci. U.S.A. 86, 3867-3871 (1989)

7. Tilney, L.G. \& Portnoy, D.A. J. Cell Biol. 109, 1597 1608 (1989).

8. Tilney, L.G. et al. J. Cell Biol. (in the press).

9. Theriot, J.A., Mitchison, T.J., Tilney, L.G. \& Portnoy, D.A. Nature 357, 257-259 (1992).

10. Helie, P., Morin, M., Jacques, M. \& Fairbrother, J.M. Infect. Immun. 59, 814-821 (1991). 